\title{
PAPERS
}

\section{What makes the dyspeptic patient feel ill? A cross sectional survey of functional health status, Helicobacter pylori infection, and psychological distress in dyspeptic patients in general practice}

\author{
A O Quartero, M W M Post, M E Numans, R A de Melker, N J de Wit
}

\begin{abstract}
Background-Dyspepsia is prevalent in about $30 \%$ of the general population in Europe, but only $25 \%$ of people with complaints consult their general practitioner. Aims-To study the relation between the severity of dyspeptic complaints and the health status of patients presenting to the general practitioner; and the relation with patient characteristics, Helicobacter pylori infection, and psychological distress. Methods-A cross sectional, general practice based survey of 360 unselected primary care dyspeptic patients from 92 general practices in The Netherlands was conducted. Symptom severity was measured using a validated symptom score, $H$ pylori using a whole blood test, and psychological distress using the GHQ-12 test. Functional health status was assessed using the COOP/Wonca charts. Results-Symptoms lasting more than three months and presence of relevant psychological distress were both associated with higher levels of dyspepsia. $H$ pylori infection, frequency of symptoms, and age had no influence on dyspepsia severity. Severity of dyspepsia and psychological distress, but not $H$ pylor $i$ infection or duration of symptoms, affected health status univariately. Dyspepsia correlated with general health, daily activities, and social activities. In logistic modelling, health status was far better predicted by psychological distress than by dyspepsia severity.

Conclusion-The relation between dyspeptic symptom severity and health status is limited. H pylori infection relates neither to functional health status, nor to intensity of dyspepsia. Psychological distress is a major determinant of impaired health of dyspeptic patients in general practice and may be the clue to improvement of health status in many dyspeptic patients.

(Gut 1999;45:15-19)
\end{abstract}

Keywords: dyspepsia; health status; general practice; Helicobacter pylori; psychological distress
Dyspepsia, a common disorder throughout Europe, is prevalent in about $30 \%$ of the general population. ${ }^{1}$ It is defined as recurrent or persistent pain or discomfort in the upper abdominal region, with or without concomitant symptoms like postprandial fullness, bloating, nausea, vomiting, heartburn, or regurgitation. ${ }^{2}$ Only $25 \%$ of people with complaints consult their general practitioner (GP). ${ }^{3}$ Symptom severity, frequency, and duration (chronic illness) of episodes, fear of the presence of a serious disease, and minor psychiatric disturbance were shown to be related to consultation behaviour of dyspeptic patients and are factors that might affect health status. $^{4-6}$ Though quality of life in relation to dyspepsia has been the subject of a number of studies, little is known about the health status of dyspeptic patients in general practice and the interrelation with psychological distress. ${ }^{7}$

Various researchers point out that psychological factors like neuroticism, anxiety, and depression are more prominent in functional and in organic dyspepsia patients, than in healthy people. ${ }^{8} 9$ Stressful life events are associated with dyspepsia. ${ }^{11}{ }^{11}$ Hypochondriasis, depression, and hysteria were more prevalent among patients with irritable bowel syndrome (IBS) and dyspepsia, but major personality disorders were found to be equally distributed among patients with functional and organic dyspepsia. ${ }^{12}$

According to these findings, it is likely that a subset of dyspeptic patients has primarily psychological factors that cause functional dyspepsia and reflux disease. ${ }^{13}$ Even in peptic ulcer disease, the role of stress as a copathogen next to Helicobacter pylori cannot be excluded. ${ }^{14} \mathrm{On}$ the one hand, psychological factors could be a cause of (functional) dyspepsia through their influence on bowel function and/or perception of symptoms, but on the other hand, dyspeptic symptoms could also directly or indirectly (worries about serious conditions) lead to psychological disturbance. ${ }^{15}$

Abbreviations used in this paper: GHQ, General Health Questionnaire; GP, general practitioner; IBS, irritable bowel syndrome. 
In general practice, the majority of patients with dyspepsia have no organic disease. ${ }^{16}$ Health status is related to $H$ pylori infection in patients with peptic ulcers, ${ }^{17}$ but no data are available on the relation between $H$ pylori and health status of patients with functional dyspepsia.

We aimed our research at the dyspeptic patients presenting in general practice, with no diagnostic differentiation, and used a validated dyspepsia symptom score and health status measurement. In a cross sectional study we sought answers to the following questions: Is symptom severity associated with the health status of dyspeptic patients in an unselected general practice population? What is the contribution of psychological distress, $H$ pylori infection, and the duration and frequency of dyspeptic episodes to symptom severity and health impairment?

\section{Methods}

DESIGN

In a cross sectional survey, 92 GPs in The Netherlands recruited 360 patients between June 1996 and April 1997. The study protocol used was approved by the local University Hospital Ethics Committee.

\section{PATIENTS}

The patients included in the survey were all between 18 and 70 years of age, and all had upper abdominal complaints lasting more than two weeks. All agreed to partake by signing informed consent forms. Patients who were pregnant or lactating, patients with a history of surgical procedures on the gastrointestinal tract (with the exception of appendectomy and inguinal hernia repair), and patients with severe comorbidity were excluded.

\section{INSTRUMENTS}

On inclusion, the GP assessed dyspepsia severity, and each patient completed a symptom questionnaire, a functional health status questionnaire, and a self rating scale on psychological distress. $H$ pylori infection was tested by the practice assistant.

An eight item symptom questionnaire was used to assess the severity of the dyspeptic complaints. The questionnaire, which scores epigastric pain, burping/belching, heartburn, bloating, flatulence, sour taste, nausea, and offensive breath (halitosis) on a five point Likert scale, is quick and efficient for assessment in daily practice and has face validity. Two validation studies in outpatient departments and in primary care showed that results are reproducible, discriminative, and sensitive to change. ${ }^{18} 19$ The scale gives a minimum score of eight and a maximum score of 40; a higher score indicates more severe complaints. The COOP/Wonca charts for measuring health status, designed and published in the United States in 1987, represent the patient's physical, social, and work performance ("functional health status") in six dimensions on a five point ordinal scale. ${ }^{20}$ Each of the six charts (general health, daily activities, social activities, physical fitness, feelings, and change of health) shows five levels, each depicted by a drawing; patients are asked to select one drawing from each chart to describe his or her status during the preceding two weeks. Developed to give GPs an instrument for assessing a wide range of patient health parameters, these charts are an easy, well validated and clinically accepted tool. ${ }^{21}$ The COOP/Wonca charts were translated, validated, and published in The Netherlands in 1992. ${ }^{22}$

The presence of psychological distress was assessed using the Dutch edition of the 12 item version of the General Health Questionnaire (GHQ-12). This questionnaire measures common disorders such as depression and anxiety, and is designed to function in open and general practice populations. It is well accepted and very easy to use as a self rating tool. Relevant psychological distress was defined as a score of three or more on the GHQ-12, as suggested in the manual. ${ }^{23}$

For diagnosis of infection with $H$ pylori, a simple whole blood serological test (BM-Test Helicobacter pylori, Roche, Switzerland) was used. Recent validation against endoscopy based tests showed acceptable performance. ${ }^{24}$

\section{DATA ANALYSIS AND STATISTICS}

Data for analysis were entered into SPSS for Windows version 6.1.3. Firstly, the relation between dyspepsia severity scores and $H$ pylori prevalence, age and sex, frequency and duration of dyspeptic episodes, and psychological distress was tested. Multiple regression analysis was used with the dyspepsia score as dependent variable, and $H$ pylori infection and possible confounders as determinants. The results indicate the correlation between $H$ pylori and the intensity of dyspeptic symptoms, corrected for the other variables included in the analysis. ${ }^{25}$ Secondly, health status scores were calculated for these variables, and tested for significance using the Mann-Whitney test. The health status scores were compared with an age matched Dutch population. ${ }^{22}$ Spearman rank correlations between dyspepsia scores, COOP/Wonca scores, and GHQ scores were then calculated. The COOP/Wonca functional health dimensions that significantly related to the dyspepsia score (coefficient greater than 0.20 ) were used in multivariate logistic analyses to check the independent influence of dyspepsia on health status. For this purpose, the COOP/Wonca charts were dichotomised using the median as cut off point. Predictors were sex, age, $H$ pylor $i$ infection, symptom severity (dyspepsia score), duration and frequency of episodes, and psychological distress.

The dyspepsia score, age, and psychological distress score were standardised for easier interpretation of the outcome (after standardisation, variables have a mean value of zero and a standard deviation of one). Stepwise backward logistic regression analyses were performed, with a forced entry of dyspepsia as final variable to study the exclusive effect of dyspepsia after controlling for all other variables. For exclusion from the model the 10\% significance level was used. ${ }^{25}$ 
Table 1 Baseline characteristics of unselected dyspeptic patients in general practice $(n=353)^{\star}$

\begin{tabular}{|c|c|c|}
\hline Mean (SD) age & 43.8 & (13.4) \\
\hline Mean (SD)dyspepsia score $†$ & 16.8 & $(4.06)$ \\
\hline No $(\%)$ of men & 164 & $(47)$ \\
\hline No (\%) with $H$ pylori infectiol & 90 & (26) \\
\hline No (\%) with $>3$ months' duration of symptoms & 171 & $(48)$ \\
\hline No (\%) with previous episodes of dyspepsia & 256 & (73) \\
\hline $\begin{array}{l}\text { No (\%) with relevant psychological distress } \\
\text { score on GHQ- } 12 \ddagger\end{array}$ & 158 & $(45)$ \\
\hline
\end{tabular}

ॠData on functional health status of seven patients were missing or incomplete, and were excluded from analysis.

+Validated eight item questionnaire on a five point Likert scale, range $9-34$.

$\ddagger \mathrm{n}=345$; data on psychological distress of eight analysed patients were missing or incomplete.

Table 2 Association of Helicobacter pylori with intensity of dyspepsia, corrected for possible confounders; results of multiple linear regression *

\begin{tabular}{lll}
\hline Variable & Beta & Significance \\
\hline H pylori infection & 0.05 & 0.34 \\
Age & -0.09 & 0.09 \\
Female sex & 0.07 & 0.16 \\
Symptoms >3 months & 0.11 & 0.03 \\
Previous episodes of dyspepsia & 0.03 & 0.59 \\
Relevant psychological distress & 0.23 & $<0.001$ \\
\hline
\end{tabular}

*In a stepwise backward model, only symptoms for longer than three months (beta $=0.12, \mathrm{p}=0.03$ ) and relevant psychological stress (beta $=0.24, \mathrm{p}=0.000)$ remain associated with a higher level of dyspepsia.

Variables that were significant predictors of health status in the model, plus those of possible biological importance for the relation between dyspepsia and the health dimension were checked for interaction effects with dyspepsia on the dependent variable.

\section{Results}

Table 1 presents baseline characteristics of the study group. Prevalence of $H$ pylori infection in the study population was $26 \%$ ( $95 \%$ confidence interval (CI): 21.2 to 30.2 ). Relevant psychological distress (GHQ-12 score of three or more) was present in $45 \%$ of the study population. Patients who had symptoms for more than three months and patients with relevant psychological distress had higher scores for dyspepsia. Infection with $H$ pylori showed no relation with symptom severity, and neither did age and frequency of dyspeptic complaints (table 2). Although women had higher dyspepsia scores than men $(17.2$ versus $16.3, p=0.04)$, sex lost its significance in multivariate analysis $(\mathrm{p}=0.16)$
In univariate comparison, a high (above median) level of dyspepsia severity and presence of relevant psychological distress was associated with greater impairment of general health, daily activities, and social activities. Psychological distress was also associated with impairment of feelings. Female dyspeptic patients suffered more from their complaints than male patients with respect to physical fitness and daily and social activities. Patients who had dyspeptic complaints for less than three months perceived a greater decrease in health than patients with longer lasting problems. Compared with an age matched study among the open population in The Netherlands, dyspeptic patients had a poorer health status than the general population (table 3).

Correlations between dyspepsia and each of the six health status dimensions were generally low: correlation coefficients above 0.20 were found for general health $(0.26, \mathrm{p}<0.001)$, daily activities $(0.31, \mathrm{p}<0.001)$, and social activities (0.26, $\mathrm{p}<0.001)$. Psychological distress correlated more strongly with these health status dimensions: $0.42,0.57$, and 0.54 for general health, daily activities, and social activities respectively (all $\mathrm{p}<0.001$ ). The strongest correlation found was between psychological distress and feelings (0.68, $\mathrm{p}<0.001$ ).

Dyspepsia proved to be a significant predictor of health status dimensions selected for logistic regression. In all instances, however, psychological distress had at least twice as much impact on functional health status as dyspepsia (table 4). Because of the strong relation between dyspepsia and psychological distress (table 2) and the interaction of these two variables in logistic regression, the impact of psychological distress was explored further. In patients with low psychological distress scores, the influence of dyspepsia severity on general health remained stable, and the predictive value of psychological distress disappeared; in patients with high psychological distress scores, the reverse happened.

$H$ pylori infection did not influence functional health: either bivariate or multivariate.

Table 3 Functional health status of dyspeptic patients in general practice, and influence of possible confounding factors ( $n=353$ )

\begin{tabular}{|c|c|c|c|c|c|c|c|c|c|c|c|c|c|}
\hline \multirow{3}{*}{$\frac{\text { Variable }}{\text { Mean (SD) }}$} & \multirow{3}{*}{ Stratum } & \multicolumn{12}{|c|}{ Functional health status with $p$ value of difference } \\
\hline & & \multicolumn{2}{|c|}{ General health } & \multicolumn{2}{|c|}{ Daily activities } & \multicolumn{2}{|c|}{ Social activities } & \multicolumn{2}{|c|}{ Physical fitness } & \multicolumn{2}{|c|}{ Feelings } & \multicolumn{2}{|c|}{ Change in health } \\
\hline & & 3.6 & $(0.8)$ & 2.4 & $(1.1)$ & 1.9 & $(1.1)$ & 2.3 & $(1.0)$ & 2.3 & $(1.2)$ & 3.1 & $(0.8)$ \\
\hline & $\leqslant 16.0^{\star}$ & 3.5 & & 2.2 & & 1.7 & & 2.2 & & 2.1 & & 3.1 & \\
\hline Dyspeptic symptom severity & $>16.0$ & 3.7 & 0.002 & 2.7 & 0.000 & 2.1 & 0.002 & 2.3 & NS & 2.4 & NS & 3.1 & NS \\
\hline \multirow[t]{2}{*}{ Sex } & Male & 3.5 & & 2.2 & & 1.8 & & 2.1 & & 2.2 & & 3.2 & \\
\hline & Female & 3.6 & NS & 2.5 & 0.03 & 2.0 & 0.04 & 2.4 & 0.009 & 2.4 & NS & 3.0 & 0.05 \\
\hline \multirow{2}{*}{ H pylori infection } & Positive & 3.6 & & 2.3 & & 1.8 & & 2.2 & & 2.1 & & 3.0 & \\
\hline & Negative & 3.6 & NS & 2.4 & NS & 1.9 & NS & 2.3 & NS & 2.3 & NS & 3.1 & NS \\
\hline \multirow{2}{*}{ Duration of dyspeptic symptoms } & $0.5-3 \mathrm{mth}$ & 3.6 & & 2.4 & & 1.9 & & 2.2 & & 2.3 & & 3.2 & \\
\hline & $>3 \mathrm{mth}$ & 3.6 & NS & 2.4 & NS & 1.9 & NS & 2.3 & NS & 2.3 & NS & 3.0 & 0.01 \\
\hline \multirow[t]{2}{*}{ Frequency of dyspeptic symptoms } & 1st episode & 3.6 & & 2.4 & & 1.9 & & 2.1 & & 2.2 & & 3.2 & \\
\hline & Previous episodes & 3.6 & NS & 2.4 & NS & 1.9 & NS & 2.3 & NS & 2.3 & NS & 3.1 & NS \\
\hline \multirow{2}{*}{ Psychological distress $\dagger$} & $\mathrm{GHQ}=<2$ & 3.3 & & 1.9 & & 1.4 & & 2.3 & & 1.7 & & 3.0 & \\
\hline & $\mathrm{GHQ}>2$ & 3.9 & 0.000 & 2.9 & 0.000 & 2.4 & 0.000 & 2.3 & NS & 3.0 & 0.000 & 3.2 & NS \\
\hline Open population ${ }^{22}$ & & 2.4 & & 1.5 & & 1.4 & & 1.9 & & 1.7 & & 2.9 & \\
\hline
\end{tabular}

* Median score of dyspepsia symptom severity.

$\dagger \mathrm{n}=345$; data on psychopathology of eight analysed patients were missing or incomplete. 
Table 4 Logistic regression analysis predicting three dimensions of health status; only the remaining variables are listed

\begin{tabular}{lccll}
\hline Variable & $B$ & $S E$ & p Value & OR \\
\hline A: Model predicting moderate/severe & impairment & of & general health & \\
Dyspepsia & 0.47 & 0.14 & $<0.001$ & 1.61 \\
Psychological distress & 0.98 & 0.17 & $<0.001$ & 2.67 \\
Sex & 0.45 & 0.25 & 0.069 & 1.57 \\
C & -0.03 & 0.39 & 0.93 & \\
B: Model predicting moderate/severe & impairment of daily & activities & & \\
Dyspepsia & 0.55 & 0.14 & $<0.001$ & 1.73 \\
Psychological distress & 1.30 & 0.17 & $<0.001$ & 3.67 \\
C & 0.06 & 0.13 & 0.66 & \\
C: Model predicting moderate/severe & impairment of social activities & & \\
Psychological distress & 1.15 & 0.16 & $<0.001$ & 3.17 \\
Dyspepsia & 0.37 & 0.13 & 0.004 & 1.45 \\
Frequency & 0.31 & 0.19 & 0.098 & 1.36 \\
C & -0.41 & 0.37 & 0.28 & \\
\hline
\end{tabular}

Model A correctly predicted the outcome for $71 \%$ of all patients; there was significant interaction between dyspepsia score and psychological distress $(p=0.03)$.

Model B correctly predicted the outcome for $72 \%$ of all patients.

Model C correctly predicted the outcome for $70 \%$ of all patients.

$\mathrm{SE}$, standard error; OR, odds ratio.

\section{Discussion}

In this study, the relation between dyspeptic complaints and six dimensions of functional health was assessed using a cross sectional survey of dyspeptic patients consulting their GP. Results showed that dyspepsia has a clear but limited influence on health status. Furthermore, psychological distress was shown to be a more powerful predictor of the health status of dyspeptic patients in general practice, especially in patients with higher levels of psychological distress. In general, infection with $H p y$ lori was not associated with severity of dyspeptic complaints or health status; $H$ pylori prevalence was similar to that found in a group of asymptomatic Dutch labourers. ${ }^{26}$

The cross sectional design of the study limits interpretation of the results. Firstly, no conclusions can be drawn regarding the causal relation of any association found in this survey; and secondly, the findings can only be generalised towards dyspeptic patients consulting their GP. We were not able to include a control group of non-dyspeptic consulters or dyspeptic non-consulters, which might have added useful information to the results of the study. The health status of non-consulting dyspeptic patients can be either better-no treatment or self care is sufficient to relieve symptoms-or worse than that of consulting dyspeptic patients: reasons not to consult can be fatalistic views on the nature of the complaint. ${ }^{27}$ Obviously, people who fear that they have cancer are likely to feel very unhealthy.

Peptic ulcers will be present in a small subgroup of the study population (approximately $10 \%$ or less). With these patients, it is likely that $H$ pylori infection is positively associated with both symptom severity and health status. We deliberately chose, however, to study all general practice dyspeptic patients. No differentiation by diagnosis or forced diagnostic work up, which could have caused selection at inclusion, was done.

There is considerable experience in using the COOP/Wonca charts for assessment of functional health status in research and clinical practice, proving them to be very suitable for general practice purposes. The charts, though criticised for lack of discriminative ability, ${ }^{28}$ which might lead to underestimation of any correlation found, gave a good representation of health status at many locations across the globe. ${ }^{29}$ Using the COOP/Wonca charts as the dependent variable in logistic regression has been done before ${ }^{30}$; we simplified our findings to the risk of being "mildly" or "more severely" impaired by a certain independent variable. One advantage of this approach is that the contributions of the different independent variables are compared and interpreted more easily. From our results it appears that general health and daily activities are the health status dimensions most impaired by dyspepsia. This observation is supported by the literature: Garrat et al found a comparable correlation between dyspepsia and similar health status dimensions using a different generic instrument-the SF-36. ${ }^{31}$ Talley et al found that patients with functional dyspepsia have specific impairment of daily activities and less limitation of physical functioning. ${ }^{32}$

Dyspeptic patients are no different to other general practice patients when psychological distress scores are compared: relevant distress appears to be present in $45 \%$ of the consulting patients compared with $25-30 \%$ of the general population. ${ }^{23}$ Similar rates of psychological distress are found in general practice sinusitis patients. ${ }^{33}$ Furthermore, stratification for psychological distress showed that general health is associated either with psychological factors (for patients with GHQ scores above two) or with severity of dyspeptic symptoms (for patients without relevant psychological distress), but not both at the same time. In the latter group, which comprises $55 \%$ of primary care dyspeptic patients, psychological factors play no role; apparently, these patients have no major worries about the nature of their complaints. For the first group of patients, distress arises in some individuals through anxiety about the seriousness of disease irrespective of symptom severity ${ }^{13}$; in other patients, possibly brought on by altered vagal tone, distress may induce gastrointestinal dysmotility and dyspeptic symptoms. Psychological factors predicted a substantial proportion of the variance in dyspeptic symptoms of patients who had to perform stress tasks, ${ }^{34}$ where almost $40 \%$ of patients with functional dyspepsia suffer from delayed gastric emptying. ${ }^{35}$ Psychological distress may also lower the threshold of visceral pain perception or affect the coping skills of dyspeptic patients. ${ }^{36} \mathrm{~A}$ stronger tendency to somatise psychological problems was found as a distinctive feature of patients with functional dyspepsia and may be another major route to the origin of dyspeptic complaints. ${ }^{37} 38$ Patients with functional dyspepsia experience a lower degree of ability to influence their situation at work than healthy reference subjects; men especially feel more psychological demands and less social support than do reference subjects. ${ }^{39}$ With these patients, even if dyspepsia is present, mental or emotional distress is obviously a much stronger reason for poor health than the abdominal discomfort. In fact, in The Netherlands, it is shown that women and divorced, unemployed, or incapacitated 
people of both sexes all have increased levels of psychological distress, and a higher chance of visiting the GP. This is irrespective of objective physical health but is clearly related to subjective perception of poor health. ${ }^{4}$ A recent study confirmed that dyspeptic subjects in general have higher levels of psychological distress, whether or not they consult a GP. Patients who visit the GP as compared with non-consulters have more severe and longer lasting symptoms and are more likely to be female. ${ }^{40}$

This might be the clue to the motivation behind the majority of patients presenting with dyspeptic symptoms in general practice: their perception of ill health is basically related to their psychological distress. Health beliefs and help seeking behaviour clearly play a critical role in this process. The role of the GP dealing with patients with a high likelihood of psychological distress should therefore not be primarily directed towards relief of dyspeptic symptoms, to $H$ pylori eradication, or to drug prescription, but towards relief of anxiety and depressive disorders - a job that no specialist performs better than the GP. ${ }^{41}$

1 Knill-Jones RP. Geographical differences in the prevalence of dyspepsia. Scand $\mathcal{F}$ Gastroenterol 1991;26(suppl 182):17-

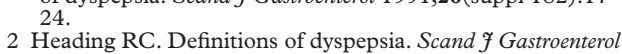
1991;26(suppl 182):1-6.

3 Jones R, Lydeard S. Prevalence of symptoms of dyspepsia in the community. BMF 1989;298:30-2.

4 Verhaak PFM. Determinants of the help-seeking process: Goldberg and Huxley's first level and first filter. Psychol Med 1995;25:95-104.

5 Talley NJ, Zinsmeister AR, Schleck CD, et al. Dyspepsia and dyspepsia subgroups: a population-based study. Gastroendyspepsia subgroups: a populd
terology 1992;102:1259-68.

6 Lydeard S, Jones R. Factors affecting the decision to consult with dyspepsia: comparison of consulters and nonconsulters. $\mathcal{F} R$ Coll Gen Pract 1989;39:495-8.

7 Wilhelmsen I. Quality of life in upper gastrointestinal disorders. Scand $\mathscr{f}$ Gastroenterol 1995;30(suppl 211):21-5.

8 Talley NJ, Fung LH, Gilligan IJ, et al. Association of anxiety, neuroticism, and depression with dyspepsia of unknown cause. A case control study. Gastroenterology 1986;90:88692.

9 Wilhelmsen I, Bakke A, Tangen Haug T, et al. Psychological adjustment to illness scale (PAIS-SR) in a Norwegian material of patients with functional dyspepsia, duodenal ulcer, and urinary bladder dysfunction. Scand $\mathcal{f}$ Gastroenterol 1994;29:611-17.

10 Talley NJ, Piper DW. Major life event stress and dyspepsia of unknown cause: a case control study. Gut 1986;27:127-34.

11 Tangen Haug T, Wilhelmsen I, Berstad A, et al. Life events and stress in patients with functional dyspepsia compared with patients with duodenal ulcer and healthy controls. with patients with duodenal ulcer and

12 Talley NJ, Phillips SF, Bruce B, et al. Relation among personality and symptoms in nonulcer dyspepsia and irritable

13 Wiklund I, Butler-Wheelhouse P. Psychological factors and their role in symptomatic gastroesophageal reflux disease and functional dyspepsia. Scand $\mathcal{F}$ Gastroenterol 1996; 31 (suppl 220): $94-100$.

14 Levenstein S. Stress and peptic ulcer: life beyond helicobacter. BMF 1998;316:538-41.

15 Drossman DA. The role of psychosocial factors in gastrointestinal illness. Scand f Gastroenterol 1996;31(suppl 221):1-4.
16 Heikkinen M, Pikkarainen P, Takal J, et al. Etiology of dyspepsia; four hundred unselected consecutive cases in general practice. Scand f Gastroenterol 1995;30:519-23.

17 Wilhelmsen I. Quality of life and Helicobacter pylori eradication. Scand $\mathcal{f}$ Gastroenterol 1996;31 (suppl 221):18-20.

18 Veldhuyzen van Zanten SJO, Tytgat KMAJ, Pollak PT, et al. Can severity of symptoms be used as an outcome measure in trials of non-ulcer dyspepsia and Helicobacter pylori associated gastritis? f Clin Epidemiol 1993;46:273-9.

19 Kuykendall DH, Rabeneck L, Campbell CJM, et al. Dyspepsia: how should we measure it? f Clin Epidemiol 1998;51:99-106.

20 Scholten JHG, van Weel C. Functional status assessment in family practice: the Dartmouth COOP Functional Health Assessment Charts/WONCA. Lelystad: Meditekst, 1992.

21 Nelson EC, Wasson JH, Johnson DJ, et al. Dartmouth COOP Functional Health assessment charts: brief measures for clinical practice. In: Spilker B, ed. Quality of life and pharmacoeconomics in clinical trials. 2nd edn. Philadelphia: Lippincott-Raven, 1996

22 Weel C van, König-Zahn C, Touw-Otten FWMM, et al. Measuring functional health status with the COOP/Wonca Charts: a manual. Groningen: Northern Centre for Health Care Research, University of Groningen, 1992; ISBN 90 72156331.

23 Koeter MWJ, Ormel J. General Health Questionnaire. Lisse, Swets en Zeitlinger BV, 1991.

24 Jones R, Phillips I, Felix G, et al. An evaluation of near-patient testing for Helicobacter pylori in general practice. Aliment Pharmacol Ther 1998;12:641-5.

25 Altman DG. Practical statistics for medical research. London: Chapman and Hall, 1991.

26 Schlemper RJ, Werf SDJ vd, Vandenbroucke JP, et al. Nonulcer dyspepsia in a Dutch working population and Helicobacter pylori. Arch Int Med 1995;155:82-7.

27 Delaney BC. Why do dyspeptic patients over the age of 50 consult their general practitioner? A qualitative investigation of health beliefs relating to dyspepsia. Br 7 Gen Pract 1998;48:1481-5.

28 Essink-Bot ML, Krabbe PFM, Bonsel GJ, et al. An empirical comparison of four generic health status measures. Med Care 1997;35:522-37.

29 Westbury RC, Rogers TB, Briggs TE, et al. A multinational study of the factorial structure and other characteristics of the Dartmouth COOP Functional Health Assessment charts/WONCA. Fam Pract 1997;14:478-85.

30 Jaffe A, Froom J, Galambos N. Minor depression and functional impairment. Arch Fam Med 1994;3:1081-6.

31 Garrat AM, Ruta DA, Russell I, et al. Developing a condition-specific measure of health for patients with dyspepsia and ulcer-related symptoms. F Clin Epidemiol 1996; 49:565-71.

32 Talley NJ, Weaver AL, Zinsmeister AR. Impact of functional dyspepsia on quality of life. Dig Dis Sci 1995;40:584-9.

33 Stalman WAB. Management of sinusitis-like complaints in general practice [thesis]. Utrecht: Utrecht University, 1997.

34 Haug TT, Svebak S, Hausken T, et al. Low vagal activity as mediating mechanism for the relationship between personality factors and gastric symptoms in functional dyspepsia.

35 Quartero AO, Wit de NJ, Lodder AC, et al. Disturbed solidphase gastric emptying in functional dyspepsia. Dig Dis Sci 1998;43:2028-33.

36 Holtmann G, Goebell H, Jockenhoevel F, et al. Altered vagal and intestinal mechanosensory function in chronic unexplained dyspepsia. Gut 1998;42:501-6.

37 Haug TT, Wilhelmsen I, Ursin H, et al. What are the real problems for patients with functional dyspepsia? Scand $\mathcal{F}$ Gastroenterol 1995;30:97-100.

38 Wilhelmsen I, Tangen Haug T, Ursin H, et al. Discriminant analysis of factors distinguishing patients with functional dyspepsia from patients with duodenal ulcer. Dig Dis Sci 1995:40:1105-11.

39 Westenberg L, Theorell T. Working conditions and family situation in relation to functional gastrointestinal disorders. The Swedish dyspepsia project. Scand $\mathcal{F}$ Prim Health Care 1997;15:76-81.

40 Talley NJ, Boyce P, Franz CP, et al. Dyspepsia and health care seeking in a community; how important are psychological factors? Dig Dis Sci 1998;43:1016-22.

41 Friedli K, King MB, Lloyd M, et al. Randomised controlled assessment of non-directive psychotherapy versus routine general-practitioner care. Lancet 1997;350:1662-5. 